\title{
Tl e eficiência organizacional: um estudo no setor brasileiro de bens de capital mecânicos com foco em micro, pequenas e médias empresas
}

\author{
Cesar Alexandre de Souza ${ }^{a *}$, Giuseppe Arpino ${ }^{\mathrm{b}}$ \\ a*calesou@usp.br, FEA/USP, Brasil \\ bgiuseppe@arpifriomaquinas.com.br, FEA/USP, Brasil
}

\begin{abstract}
Resumo
Uma das medidas para o sucesso do uso da tecnologia da informação (Tl) é seu impacto na performance das empresas. Muitos estudos têm sido realizados analisando a influência da $\mathrm{Tl}$ no resultado, especialmente em empresas de grande porte. Este trabalho tem como objetivo analisar a relação entre investimentos em $\mathrm{Tl}$ e eficiência organizacional com foco em empresas de micro, pequeno e médio porte. Para isso, foram identificados fatores críticos de sucesso para o desempenho de empresas industriais, sendo em seguida desenvolvido um modelo para realização de análise envoltória de dados (DEA - Data Envelopment Analysis) em dois estágios. A pesquisa de campo foi realizada com empresas ligadas à Associação Brasileira das Indústrias de Máquinas e Equipamentos (Abimaq). Dentre os resultados destacam-se a maior capacidade das empresas de pequeno porte em converter o uso da Tl em eficiência operacional e a maior capacidade das empresas maiores em converter fatores críticos de sucesso em rentabilidade.

Palavras-chave

Tecnologia da informação. Informatização. Manufatura. Gestão de micro, pequenas e médias empresas. Análise Envoltória de Dados.
\end{abstract}

\section{Introdução}

Há várias razões que justificam o estudo da influência do uso da tecnologia da informação (TI) na eficiência das empresas. Em particular, isso é importante nas empresas de manufatura brasileiras, onde a necessidade de investimentos em $\mathrm{Tl}$ adveio principalmente de atrasos tecnológicos causados por particularidades econômicas do país. Com a recente recuperação econômica brasileira tornou-se mister recobrar o atraso para tentar equilibrar a competitividade externa, investindo tanto em $\mathrm{Tl}$ aplicada à gestão e à produção, como em técnicas modernas de manufatura, tais como o Just in Time (JIT) e os Flexible Manufacturing Systems (FMS), entre outras. Portanto, avaliar de forma correta a aplicação dos recursos de $\mathrm{Tl}$ tem sido uma preocupação constante para as empresas industriais dos diversos setores e portes, mais especialmente nas micro, pequenas e médias empresas (SOUZA et al., 2005).
Trabalhos acadêmicos que retratem a realidade nacional das empresas de manufatura, especialmente de micro, pequeno e médio porte, serão, portanto, de grande valia para as estratégias de penetração de mercado das firmas produtoras de software e para as próprias empresas usuárias, que poderão utilizar seus resultados e conclusões como benchmarking para seus processos de informatização, aspectos que justificam a elaboração do presente estudo.

Este trabalho tem por objetivo analisar a relação entre investimentos em Tl e eficiência organizacional em empresas brasileiras do setor de bens de capital, procurando identificar aspectos que distinguem as empresas que conseguem obter melhores resultados pelo uso da Tl. Para Mello (2004), no Brasil o setor é composto por cerca de 4.000 empresas, sendo que $65 \%$ dessas são de pequeno porte ou microempresas, 25\% são de médio porte e 10\% são grandes empresas. 
Inicialmente é apresentado um breve referencial teórico sobre estudos envolvendo $\mathrm{Tl}$ e desempenho empresarial, destacando-se o uso da técnica de análise de dados proposta (análise envoltória de dados ou DEA - Data Envelopment Analysis) e relatando estudos realizados na área de manufatura. A relação da técnica com o objetivo do trabalho é então explicitada, e é apresentado o modelo de pesquisa. São então determinados os inputs e outputs do modelo, a partir da análise dos fatores críticos de sucesso e dos indicadores de desempenho para as empresas industriais, bem como os fatores organizacionais e de uso de Tl (ou informatização) a eles relacionados. A técnica DEA é aplicada em dois estágios, buscando relacionar a aplicação da Tl inicialmente a impactos nos processos operacionais das empresas e seus fatores críticos de sucesso, e, em seguida, relacionar esses impactos a resultados de desempenho financeiro da organização. Os resultados da DEA são então comparados aos fatores de informatização e características das empresas. Finalmente, os resultados são discutidos e as conclusões apresentadas.

Esse trabalho apresenta as seguintes contribuições: fornece dados sobre o grau de informatização de empresas do setor de bens de capital mecânico brasileiro; elabora um modelo relacionando a aplicação de $\mathrm{Tl}$ em empresas de manufatura ao desempenho, sugerindo medidas para os efeitos intermediários da aplicação da Tl ligadas a fatores críticos de sucesso; e relaciona a obtenção de resultados pelo uso da $\mathrm{Tl}$ a fatores de informatização.

\section{Referencial teórico}

\section{1. $\mathrm{Tl}$ e desempenho de empresas}

A partir do final dos anos 80 houve uma forte suspeita por parte de acadêmicos e empresas usuárias de que os investimentos realizados em Tl não estavam produzindo os resultados esperados. 0 chamado "paradoxo da produtividade" atraiu a atenção dos pesquisadores e profissionais de mercado, pois não se estavam encontrando evidências suficientes de que o investimento em $\mathrm{Tl}$ estivesse de fato contribuindo para ganhos concretos nas empresas. Procurando buscar indícios opostos ao paradoxo, diversas pesquisas começaram a identificar as contribuições da Tl para os resultados, especialmente em grandes empresas (HITT; BRYNJOLFSSON, 1994; RAl; PATNAYAKUNI; PATNAYAKUNI, 1997; LEE; BARUA, 1999; MAÇADA; BECKER; LUNARDI, 2005).
Um número menor de pesquisas foi realizado para verificar a existência ou não do paradoxo da produtividade em micro, pequenas e médias empresas (MPMEs), conforme atestado por Valente e Riccio (2004), visto que, até pouco tempo, os sistemas integrados de gestão eram normalmente destinados apenas a grandes corporações em razão dos elevados custos e complexidade envolvida em sua implementação. Muitas MPMEs consideram o investimento alto, desconhecem as possibilidades e dificuldades do uso da tecnologia e não sabem como avaliar as melhorias obtidas. Assim, ainda não estão devidamente esclarecidas as consequências do investimento em informatização em empresas menores, apesar das pesquisas já realizadas neste contexto (MENDES; ESCRIVÃO FILHO, 2002; BUONANNO; GRAMIGNOLI; RAVARINI, 2002; AHUMADA-LOBO, 2002; BECCHETTI, 2003).

A pesquisa de Mendes e Escrivão Filho (2002) mostrou que, diferentemente das implementações nas empresas de grande porte, algumas dificuldades enfrentadas pelas pequenas e médias empresas na implementação de ERPs nem sequer são mencionadas na literatura, e outras que aparecem repetidamente no referencial teórico não são relatadas pelas empresas pesquisadas. Buonanno, Gramignoli e Ravarini (2002) mostraram que os resultados obtidos em implantações de sistemas de informação são muito diferentes entre pequenas e médias empresas: ambas têm consciência de sua importância, mas as médias parecem ter mais recursos para investir em adoção da Tl e seu gerenciamento. Ao contrário, as pequenas empresas são caracterizadas por conhecimento técnico limitado, que parece ser a maior barreira para a implantação dos projetos de Tl. Em outro trabalho, AhumadaLobo (2002) concluiu que existe correlação positiva entre investimentos $\mathrm{em} \mathrm{Tl}$ e a produtividade em um grande número de pequenas e médias empresas espanholas. Segundo seus resultados, empresas que investem consistentemente mais em $\mathrm{Tl}$ tendem a ter maior faturamento que as que pouco investem em praticamente todos os setores estudados. Becchetti (2003) mostrou que o investimento em $\mathrm{Tl}$ em pequenas e médias empresas italianas influenciou positivamente a elaboração de novos produtos e processos, além de aumentar a capacidade produtiva, criando uma "opção de flexibilidade" em que decisões irreversíveis, como a construção de uma nova planta industrial, possam ser postergadas, possibilitando atender mais rapidamente o apetite de seus clientes por variedades e novidades. 0 autor conclui que o investimento em software aumenta a necessidade de funcionários especializados, mas por outro lado também aumenta a produtividade no trabalho. 


\subsection{Estudos sobre $T 7$ e desempenho e uso da $D E A$}

A DEA tem sido utilizada como ferramenta analítica em diversos estudos que procuram relacionar o uso de $\mathrm{Tl}$ a medidas de produtividade empresarial, oferecendo vantagens nessa aplicação (WANG; GOPAL; ZIONTS, 1997). A DEA é um método comparativo agregado para medir a produtividade relativa de unidades de análises (DMUs, ou decision making units), que transformam múltiplas entradas (inputs) em múltiplas saídas (outputs). A técnica utiliza um modelo de programação linear para construir uma unidade hipotética baseada em todas as unidades do grupo de referência. Isto é, a performance de cada DMU é medida relativamente à performance de todas as DMUs. A DMU é definida como uma empresa, departamento, divisão ou unidade administrativa, cuja eficiência está sendo avaliada. 0 conjunto de DMUs adotadas em uma análise DEA deve ter em comum a utilização dos mesmos inputs e outputs, ser homogêneo e ter autonomia na tomada de decisões. A unidade sendo avaliada pode ser julgada relativamente ineficiente se a unidade composta requer menos entradas para obter as mesmas saídas da unidade em avaliação ou julgada relativamente eficiente se a unidade composta requer as mesmas entradas da unidade em avaliação. Os resultados da DEA ajudam a identificar uma DMU relativamente ineficiente e fornecem pistas de como melhorar a produtividade dessa unidade. Segundo Siems e Barr apud Maçada, Becker e Lunardi (2005), o uso da técnica DEA possibilita: determinar quantitativamente a eficiência relativa de cada DMU, sob forma de taxas; identificar origens e quantidades de ineficiência relativa em cada uma das DMU, em qualquer de suas dimensões (input ou output); e apoiar o planejamento para maximização da eficiência de cada DMU.

Entre as vantagens da utilização da técnica nesse contexto apontadas por Wang, Gopal e Zionts (1997) estão: os dados sobre os investimentos em Tl não necessitam ser normalizados, por exemplo, dividindo o valor investido pelo faturamento ou pelo número de funcionários, como é necessário nas abordagens econométricas; a técnica não exige modelagem a priori de uma função relacionando as saídas às entradas, o que é uma vantagem no caso da análise de investimentos em $\mathrm{Tl}$, em que pouco se sabe a respeito da dinâmica da interação entre entradas e saídas no processo de obtenção de valor pelo uso da Tl; a técnica permite identificar explicitamente a eficiência do processo de transformação dos investimentos $\mathrm{em} \mathrm{Tl} \mathrm{em}$ resultados para cada unidade de análise; finalmente, como resultado das duas características anteriores, a DEA permite que se evite o problema de analisar diretamente a relação entre investimentos em $\mathrm{Tl}$ e resultados da empresa, uma vez que a empresa pode estar investindo, mas não estar usando adequadamente os sistemas (aspecto apontado como uma das causas do "paradoxo"). Assim, a técnica permite que as empresas eficientes na conversão de investimentos em $\mathrm{Tl}$ em resultados sejam diferenciadas das demais, e, em análises posteriores, essa eficiência seja relacionada a outros fatores como grau e qualidade do uso da $\mathrm{Tl}$, qualidade da gestão de $\mathrm{Tl}$, alinhamento estratégico, entre outros (e, por isso a sua adequação aos objetivos deste trabalho). Mais detalhes sobre a formulação matemática da DEA e seus aspectos computacionais podem ser obtidos em Wang, Gopal e Zionts (1997), Maçada, Becker e Lunardi (2005) e Surco e Wilhem (2004).

Wang, Gopal e Zionts (1997) ressaltam que o impacto da $\mathrm{Tl}$ sobre os resultados nas empresas pesquisadas é indireto e, consequentemente, os impactos da $\mathrm{Tl}$ devem ser observados e analisados a partir de processos de produção intermediários. Esses pontos levaram os autores a desenvolver um modelo de produção em dois estágios com o objetivo de mapear o quanto os processos intermediários afetam a eficiência das organizações. Na abordagem de Maçada, Becker e Lunardi (2005), o modelo de eficiência estrutura-se no conceito de efetividade de conversão, que pressupõe que os investimentos em $\mathrm{Tl}$, combinados com as variáveis de input (despesas com pessoal, outras despesas administrativas e despesas de internacionalização), transformam-se em output (receitas líquidas de intermediação financeira, de prestação de serviços e de operações internacionais). Similarmente ao trabalho de Wang, Gopal e Zionts (1997), os autores adotaram um modelo com dois estágios apoiados no conceito de efetividade de conversão (WEILL, 1989), que é a capacidade de converter os investimentos em $\mathrm{Tl}$ em valores que possam trazer retorno à organização. 0 primeiro estágio contemplava como entradas os investimentos em $\mathrm{Tl}$ e em pessoal e como saídas os ativos dos bancos que poderiam ser influenciados por investimentos em Tl. Essas mesmas saídas serviram como entradas para o segundo estágio, sendo as receitas financeiras consideradas como sua saída final. Essa pesquisa mostrou que bancos que investiram mais em $\mathrm{Tl}$ ganharam eficiência ao longo do tempo. 


\subsection{Estudos sobre $T 1$ e desempenho e uso da DEA na área de manufatura}

Alguns estudos têm aplicado a DEA na área de manufatura. Dasgupta, Sarkis e Talluri (1999), por exemplo, analisaram a influência do investimento em $\mathrm{Tl}$ a resultados de empresas de manufatura e de serviços. A metodologia adotada utilizou a DEA em diversas configurações, seguidas de teste estatístico que mostrou que o índice de eficiência estava negativamente correlacionado aos valores investidos em Tl. Segundo os autores, uma razão possivel para esses resultados é que, à medida que as empresas investem mais em $\mathrm{Tl}$ de gestão e de produção, existe maior necessidade de coordenar as diferentes atividades e sistemas em todas as áreas da organização, possivelmente indicando um ganho decrescente de escala na informatização. Esse resultado é importante em relação à proposta deste trabalho, uma vez que o objetivo é identificar que outros fatores, além do valor do investimento em si, permitem diferenciar empresas que convertem melhor os investimentos em $\mathrm{Tl}$ em resultados.

Petroni e Bevilacqua (2002) aplicaram a metodologia DEA para identificar as MPMEs que operam na fronteira eficiente da manufatura flexível com dados obtidos de 89 empresas da região de Brescia (Itália). 0 modelo DEA adotado utilizou as seguintes medidas de output: grau de flexibilidade de maquinário, grau de flexibilidade de processos, grau de flexibilidade de produtos, grau de flexibilidade de roteamento, grau de flexibilidade de volume, grau de flexibilidade de expansão e grau de flexibilidade de layout, diversos aspectos ligados à eficiência operacional em empresas de manufatura. Em seguida, utilizou análise discriminante para identificar dois subgrupos de manufatura flexível: empresas flexíveis voltadas à customização em massa e empresas flexíveis voltadas à inovação. 0 trabalho atingiu o objetivo de classificar as empresas segundo as melhores práticas estipuladas. Embora não especificamente ligado à avaliação do uso da $\mathrm{Tl}$, este trabalho permite perceber as possibilidades de aplicação da técnica ao utilizar uma série de variáveis ligadas aos aspectos do processo produtivo como saídas do modelo. Essa possibilidade será utilizada neste trabalho.

\section{Metodologia e modelo de pesquisa}

Os objetivos deste trabalho são analisar a relação entre investimentos em $\mathrm{Tl}$ direcionados para a gestão e produção em empresas brasileiras do setor de bens de capital, e sua eficiência organizacional, e identificar quais fatores estão associados a um melhor ou pior aproveitamento desses investimentos. 0 tipo de pesquisa realizado neste trabalho é uma combinação de pesquisa descritiva e exploratória, com método de análise quantitativo, utilizando-se a técnica DEA, que tem os detalhes de seu emprego apresentados a seguir.

\subsection{Modelo DEA}

Seguindo as considerações apresentadas por Wang, Gopal e Zionts (1997) e Maçada, Becker e Lunardi (2005), a proposta deste trabalho é desenvolver a análise da DEA em dois estágios, utilizando o grau de informatização como input e os diversos fatores críticos de sucesso para as empresas de bens de capital como outputs intermediários, associando os resultados financeiros como output final do modelo. Com isso, foi elaborado o modelo inicial de análise de eficiência de dois estágios, apresentado na Figura 1.

0 primeiro estágio do modelo será denominado de estágio de eficiência operacional e terá três entradas (despesas em $\mathrm{Tl}$, investimentos em $\mathrm{Tl}$ e outros custos da empresa) e 7 saídas (qualidade,

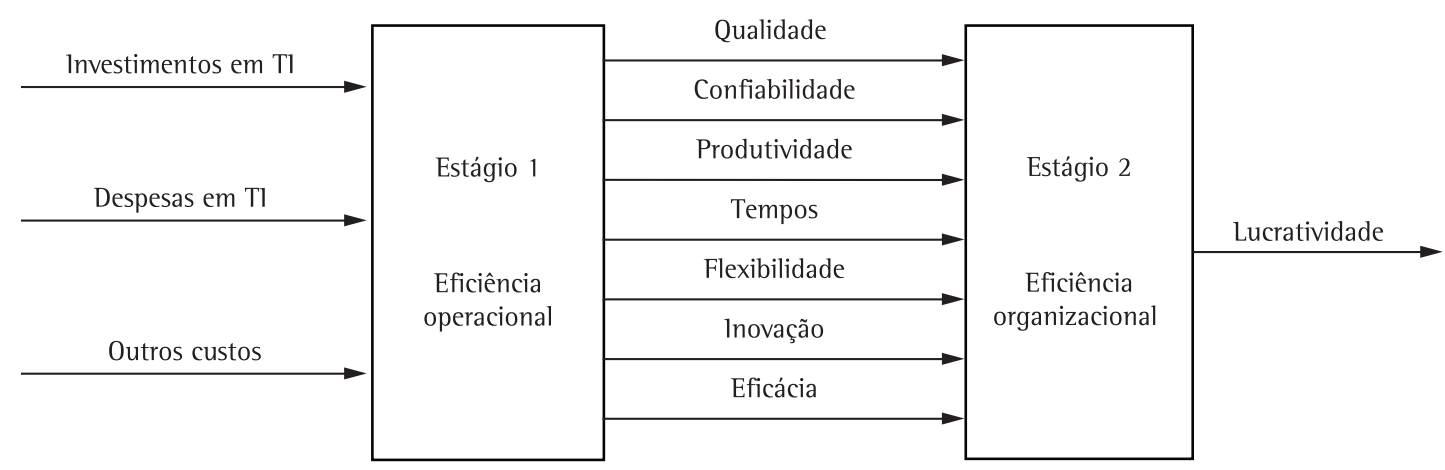

Figura 1. Modelo inicial de análise de eficiência (elaborado pelos autores). 
confiabilidade, produtividade, tempos, flexibilidade, inovação e eficácia). 0 segundo estágio, denominado estágio de eficiência em rentabilidade, utilizará as sete saídas do primeiro estágio como suas próprias entradas, de forma a verificar suas influências no output lucratividade. Nos próximos itens estão descritas cada uma das variáveis originalmente consideradas.

\subsubsection{Entradas do primeiro estágio}

Como entradas do primeiro estágio comparecem as variáveis associadas a despesas e investimentos em $\mathrm{Tl}$, ligadas à mensuração dos investimentos em $\mathrm{Tl}$ direcionados para a gestão e produção em empresas, como enunciado no objetivo do trabalho. A composição dessas variáveis, recomendadas pela bibliografia em estudos que analisaram os resultados de investimentos em $\mathrm{Tl}$, é apresentada no Quadro 1. Note que também os demais custos e investimentos estão incluídos no modelo, que pressupõe que os investimentos de $\mathrm{Tl}$ devem ser combinados aos demais inputs de produção para que se obtenham os resultados desejados (MAÇADA; BECKER; LUNARDI, 2005).

\subsubsection{Saídas do primeiro estágio}

0 primeiro estágio, denominado "eficiência operacional”, tem como saídas variáveis que representam o desempenho da empresa em uma série de aspectos importantes para o seu sucesso, mas relativos exclusivamente à operação da empresa. Essas variáveis estão ligadas à mensuração dos aspectos inerentes à eficiência organizacional, como descrito no objetivo do trabalho. Procurou-se elencar quais seriam esses aspectos analisando a literatura sobre fatores críticos de sucesso em manufatura. De acordo com Francischini (1998), os fatores críticos de sucesso são aquelas variáveis nas quais a empresa precisa ter, necessariamente, bom desempenho para ser competitiva. Com base em Muscat e Fleury (1993) e Tironi (1993) foram identificados os fatores críticos de sucesso e indicadores para sua mensuração apresentados no Quadro 2.

\subsubsection{Saídas do segundo estágio}

0 segundo estágio, denominado "eficiência organizacional", tem como saídas variáveis que representam os resultados financeiros ou de mercado decorrentes das operações da empresa, e diretamente ligadas à mensuração da eficiência organizacional, como exposto no objetivo do trabalho. Os fatores mais comumente associados a resultados finais de uma organização são relacionados a receitas, lucratividade e participação no mercado. Os demais (qualidade, confiabilidade, produtividade, tempos, flexibilidade, inovação e eficácia) denotam resultados associados aos processos intermediários que podem levar a empresa a uma redução de custos e/ou a uma maior lucratividade. No modelo, a variável lucratividade, medida como lucro na forma de porcentagem das vendas, representa a saída do segundo estágio.

\subsection{Fatores determinantes do uso adequado da $T 1$ de gestão e de produção}

Para a análise de fatores que podem estar associados a um melhor desempenho no que se refere à transformação dos investimentos em $\mathrm{Tl}$ em resultados empresariais, tanto no primeiro como no segundo estágio do modelo, foram identificados na literatura fatores ligados a características que podem ter influência no referido processo. Considerando-se os estudos pesquisados (SOUZA et al., 2005; LAGACÉ, 2000; KUDYBA; DIWAN, 2002), pôde-se identificar três fatores, descritos no Quadro 3. Esses fatores, aqui denominados fatores de informatização, foram utilizados em uma análise exploratória procurando compará-los com os resultados obtidos na análise DEA e para verificação de possíveis interações, e estão relacionados à identificação dos aspectos associados a um melhor ou pior aproveitamento dos investimentos em Tl, como exposto no objetivo do trabalho.

\subsection{Metodologia de coleta de dados, universo pesquisado e questionário utilizado}

A coleta de dados foi feita através de questionário desenvolvido em MS-Word enviado por correio eletrônico em fevereiro de 2008 para uma lista de 3.833 empresas, entre associadas e não associadas, fornecida pela Abimaq. Segundo Avellar (2007), o setor de bens de capital reúne um conjunto de fabricantes de máquinas e equipamentos e cumpre um papel determinante na difusão de novas tecnologias e como catalisador do crescimento econômico. 0 setor engloba uma diversa gama de produtos que podem ser agrupados em: bens de capital mecânicos (mecânica, equipamentos industriais, máquinas e implementos agrícolas, máquinas rodoviárias); material de transporte; e material elétrico e de comunicações. Desse universo, que é formado principalmente por micro e pequenas empresas que trabalham sob 
Quadro 1. Descrição das variáveis de input e sua composição.

\begin{tabular}{|c|c|c|}
\hline Variáveis & Composição básica & Referência \\
\hline $\begin{array}{c}\text { Despesas com Tl de gestão } \\
\text { e de produção }\end{array}$ & $\begin{array}{c}\text { Hardware, software, telecomunicações, funcionários de Tl } \\
\text { de gestão e de produção, serviços }\end{array}$ & $\begin{array}{c}\text { Rai, Patnayakuni e Patnayakuni (1997), } \\
\text { Weill (1992) }\end{array}$ \\
\hline $\begin{array}{c}\text { Investimentos em Tl de } \\
\text { gestão e de produção }\end{array}$ & Infraestrutura, arquitetura cliente-servidor, aplicativos & $\begin{array}{c}\text { Rai, Patnayakuni e Patnayakuni (1997), } \\
\text { Dasgupta, Sarkis e Talluri (1999), } \\
\text { Kudyba e Diwan (2002) }\end{array}$ \\
\hline Demais custos da empresa & Custos de mão de obra, matéria-prima e despesas administrativas & Maçada, Becker e Lunardi (2002) \\
\hline
\end{tabular}

Quadro 2. Fatores críticos de sucesso do setor de bens de capital e sua composição.

\begin{tabular}{|c|c|c|}
\hline $\begin{array}{c}\text { Fatores criticos } \\
\text { de sucesso }\end{array}$ & Indicadores de desempenho & Referência \\
\hline Qualidade & Nível de falhas, certificações & Serson (1996) \\
\hline Confiabilidade & Porcentagem de pedidos entregues com atraso, tempo de atraso médio dos pedidos & Serson (1996) \\
\hline Produtividade & Produtividade física da mão de obra, quantidade produzida por homens-hora, giro de estoque & $\begin{array}{c}\text { Corrêa e Corrêa (2005); } \\
\text { Sink e Tuttle (1993) }\end{array}$ \\
\hline Tempos & Tempo do ciclo de produção, tempo do ciclo de fornecimento, frequência de entrega & Serson (1996) \\
\hline Flexibilidade & Tempo para mudar programações, flexibilidade da mão de obra & Serson (1996) \\
\hline Inovação & $\begin{array}{c}\text { Número de novos procedimentos adotados, número de novos produtos lançados por período } \\
\text { com sucesso, redução de estoques, número de pessoas em P\&D }\end{array}$ & Sink e Tuttle (1993) \\
\hline Eficácia & Faturamento obtido/faturamento previsto, tempo real de produção/tempo esperado de produção & Serson (1996) \\
\hline Custo & Custo por hora de operação, taxa horária das instalações, custo unitário de materiais, taxa \\
horária de mão de obra & Serson (1996) \\
\hline
\end{tabular}

Quadro 3. Fatores de Informatização e sua composição.

\begin{tabular}{|c|c|c|}
\hline Fator de informatização & Composição básica & Referências \\
\hline Grau de informatização (Gl) & $\begin{array}{l}\text { Grau e extensão de uso, dependência, qualidade técnica e integração } \\
\text { dos sistemas presentes }\end{array}$ & Souza et al. (2005) \\
\hline $\begin{array}{c}\text { Grau de integração tecnológica e } \\
\text { organizacional (10) }\end{array}$ & Grau de integração organizacional e da cadeia de suprimentos & $\begin{array}{c}\text { Lagacé (2000); } \\
\text { Kudyba e Diwan (2002) }\end{array}$ \\
\hline Condições de operação (CO) & $\begin{array}{l}\text { Planejamento de } \mathrm{Tl} \text {, participação e conhecimento de funcionários } \\
\text { e executivos no planejamento de } \mathrm{Tl}\end{array}$ & $\begin{array}{l}\text { Souza et al. (2005); } \\
\text { Lagacé (2000) }\end{array}$ \\
\hline
\end{tabular}

encomenda, 80 responderam $(2,1 \%)$ até o fim da coleta em março de 2008, e dessas 28 puderam ser aproveitadas para compor o grupo testado pelo método DEA. 0 questionário foi elaborado com base nas variáveis e fatores descritos nos itens 3.1 e 3.2, de acordo com as referências bibliográficas. A partir das informações fornecidas pelas empresas estabeleceram-se os valores para cada input e output do modelo. 0 questionário é extenso para inclusão neste trabalho e pode ser obtido por solicitação aos autores, mas seus principais aspectos estão apresentados a seguir.

Em relação às entradas do primeiro estágio do modelo, para compor os valores de despesas em $\mathrm{Tl}$, foram perguntados os valores médios gastos mensalmente em manutenção de hardware e software, incluindo sistemas e equipamentos ligados à produção. 0 valor dos investimentos foi estimado a partir do número de microcomputadores e sistemas de gestão e produção instalados, conforme relatado pelas empresas. Para obter os demais custos da empresa foi solicitado que fosse informado o percentual de custos em relação ao faturamento da empresa. Sobre as saídas do primeiro estágio foram elaboradas questões com base na bibliografia e indicadores apresentados no item 3.1.2 (Quadro 2). Algumas das questões, que puderam ser utilizadas no modelo final, estão apresentadas no Quadro 4. Finalmente, para compor a saída do segundo estágio, foi perguntado às empresas o lucro de 2007 como percentual do faturamento desse mesmo ano. Em relação aos fatores de informatização (grau de informatização, grau de integração tecnológica e organizacional e condições de operação), foram utilizadas as questões desenvolvidas pelos autores apontadas no item 3.2, compostas por escalas do tipo Likert. 0 questionário foi testado previamente em três empresas, contando-se também com o auxílio de um consultor da Abimaq para refiná-lo e simplificá-lo, procurando torná-lo acessível ao público alvo da pesquisa. 
Quadro 4. Questões do questionário ligadas às saídas do primeiro estágio do modelo.

\begin{tabular}{|c|c|c|}
\hline Questões apresentadas & Indicador de desempenho pretendido & FCS \\
\hline $\begin{array}{c}\text { Indique a percepção da qualidade do(s) seu(s) produto(s) por parte dos } \\
\text { clientes (1 = baixa; 5 = alta) }\end{array}$ & Qualidade percebida & Qualidade \\
\hline Cite as certificações oficiais de qualidade obtidas pela empresa (se tiver) & Número de certificações & Qualidade \\
\hline Qual o percentual de máquinas entregues com atraso em 2007? & \% de pedidos com atraso & Confiabilidade \\
\hline $\begin{array}{c}\text { Indique a quantidade de máquinas produzidas em 2007, excluindo acessórios } \\
\text { A empresa adotou processo(s) inovador(es) durante os últimos dois anos } \\
\text { (JIT, FMS, downsizing, reengenharia, seis sigma, outros)? Se sim, quais? }\end{array}$ & Novos processos adotados por ano & Inovação \\
\hline $\begin{array}{c}\text { Número de novos produtos lançados nos últimos dois anos, não } \\
\text { considerando melhorias feitas em produtos já existentes }\end{array}$ & Número de novos produtos & Inovação \\
\hline $\begin{array}{c}\text { Número médio de funcionários trabalhando em P\&D (pesquisa e } \\
\text { desenvolvimento) em 2007 }\end{array}$ & Número de funcionários em P\&D & Inovação \\
\hline $\begin{array}{c}\text { Do tempo total para produção de um equipamento, qual o percentual } \\
\text { despendido em set-up? }\end{array}$ & $\begin{array}{c}\text { Tempo médio para mudar programação } \\
\text { de produção }\end{array}$ & Flexibilidade \\
\hline $\begin{array}{c}\text { Qual porcentagem dos funcionários da produção capazes de executar } \\
\text { todas as atividades produtivas do setor? }\end{array}$ & Flexibilidade da mão de obra & Flexibilidade \\
\hline Qual o número total de horas extras trabalhadas em 2007? & $\begin{array}{c}\text { Tempo médio real de produção/tempo médio } \\
\text { esperado de produção }\end{array}$ & Eficácia \\
\hline
\end{tabular}

\section{Resultados obtidos}

\subsection{Análise descritiva dos dados}

Como primeira etapa da análise dos dados procedeu-se a uma análise descritiva exploratória com dois objetivos: caracterizar a amostra quanto ao porte, setor e características produtivas e apresentar os dados relativos ao grau de informatização das empresas do setor de bens de capital brasileiro. A análise exploratória dos dados permitiu identificar os casos extremos (outliers) que foram retirados desde que não se tenha confirmado sua veracidade junto às empresas respondentes.

\subsubsection{Amostra obtida}

Das 80 empresas que responderam parcial ou totalmente o questionário, 23\% são microempresas, $47 \%$ são pequenas empresas, $20 \%$ são médias empresas e 10\% são grandes empresas. Tal distribuição reflete com bastante proximidade a do cadastro original. Para a divisão da amostra por porte das empresas (micro, pequena, média e grande), foi utilizada a definição baseada no número de empregados (microempresa de 1 a 9 funcionários; pequena empresa de 10 a 99; média de 100 a 499; grande empresa 500 ou mais). Por ter recebido um acompanhamento mais sistemático durante a fase de coleta de dados (com reenvio de e-mails e telefonemas pessoais), o subsetor de máquinas-ferramenta foi o que mais respondeu (47,5\%). 0 objetivo desse destaque foi o de obter uma quantidade suficiente de empresas de um único subsetor, de maneira a poder-se realizar uma comparação dentro de um subsetor específico utilizando a DEA. A Tabela 1 apresenta características das empresas da amostra e as quantidades médias de funcionários, faturamento e lucro, por porte.

\subsubsection{Despesas e investimentos em $\mathrm{Tl}$}

Na Tabela 2 estão apresentados os valores médios por porte de empresa e geral para as despesas anuais em $\mathrm{Tl}$ e para o investimento acumulado em $\mathrm{Tl}$ (calculado a partir da valoração dos equipamentos e sistemas possuídos pela empresa, a valores atuais de mercado). Os valores foram divididos pelo faturamento informado em 2007, para possibilitar a comparação (note-se que no caso dos investimentos em $\mathrm{Tl}$, não se trata do valor investido no ano de 2007, mas ao longo do tempo - a relação apenas permite tornar os investimentos comparáveis por empresa, não se tratando do investimento realizado naquele ano).

Observa-se que apesar de as despesas e investimentos aumentarem com o tamanho da empresa, a relação com o faturamento vai ficando cada vez menor. Isso explica a maior capacidade relativa das grandes empresas de gastarem mais com Tl. De qualquer forma, pode-se afirmar que a capacidade de investimento em $\mathrm{Tl}$ aumenta com o porte da empresa.

\subsubsection{Análise das atividades apoiadas por $\mathrm{Tl}$}

Para a composição do grau de informatização das empresas, um dos fatores determinantes de resultados do uso da $\mathrm{Tl}$ empregados na análise, foi perguntado às empresas quais atividades eram conduzidas com apoio de sistemas informatizados. $\mathrm{Na}$ Tabela 3 está apresentado o percentual de 
Tabela 1. Características das empresas da amostra por porte.

\begin{tabular}{ccccccc}
\hline Porte & $\mathbf{n}$ & $\%$ & $\begin{array}{c}\text { Número de funcionários } \\
\text { (média) }\end{array}$ & $\begin{array}{c}\text { Faturamento 2007 (R\$) } \\
\text { (média) }\end{array}$ & $\begin{array}{c}\text { Lucro (R\$) } \\
\text { (média) }\end{array}$ & $\begin{array}{c}\text { Lucro (\%) } \\
\text { (média) }\end{array}$ \\
\hline Micro & 18 & 22,5 & 9 & $2.684 .355,00$ & $201.327,00$ & 7,50 \\
Pequena & 38 & 47,5 & 50 & $8.333 .636,00$ & $827.530,00$ & 9,93 \\
Média & 16 & 20,0 & 148 & $55.978 .719,00$ & $5.178 .032,00$ & 9,25 \\
Grande & 8 & 10,0 & 2.325 & $820.363 .663,00$ & $54.636 .220,00$ & 6,66 \\
Geral & 80 & 100,0 & 288 & $70.617 .686,00$ & $6.249 .665,00$ & 8,85 \\
\hline
\end{tabular}

Tabela 2. Despesas e investimentos em Tl por porte e geral.

\begin{tabular}{|c|c|c|c|c|c|c|c|c|}
\hline Porte & $\mathrm{n}$ & $\begin{array}{c}\text { Despesas anuais } \\
\text { em Tl (R\$) }\end{array}$ & $\mathrm{n}$ & $\begin{array}{c}\text { Despesas anuais/ } \\
\text { faturamento } 2007 \\
(\%)\end{array}$ & $\mathrm{n}$ & $\begin{array}{c}\text { Investimento } \\
\text { acumulado em } \mathrm{Tl} \\
\text { (R\$) }\end{array}$ & $n$ & $\begin{array}{c}\text { Investimento em Tl/ } \\
\text { faturamento } 2007 \\
(\%)\end{array}$ \\
\hline Micros & 14 & $23.013,47$ & 14 & 2,8 & 17 & $48.289,65$ & 14 & 4,11 \\
\hline Pequenas & 26 & $50.502,00$ & 26 & 1,0 & 38 & $306.103,50$ & 31 & 5,38 \\
\hline Médias & 6 & $122.592,00$ & 6 & 0,5 & 16 & $954.318,19$ & 8 & 2,50 \\
\hline Grandes & 3 & $1.694 .250,00$ & 3 & 0,3 & 8 & $8.783 .322,75$ & 4 & 1,13 \\
\hline Geral & 49 & $223.109,88$ & 49 & 1,4 & 79 & $1.240 .361,14$ & 57 & 4,36 \\
\hline
\end{tabular}

Tabela 3. Grau de uso de sistemas por porte (\%).

\begin{tabular}{|c|c|c|c|c|c|c|}
\hline & Aplicativo/sistema & $\begin{array}{l}\text { Todas } \\
(\mathrm{n}=76)\end{array}$ & $\begin{array}{l}\text { Micro } \\
(\mathrm{n}=15)\end{array}$ & $\begin{array}{l}\text { Pequena } \\
(\mathrm{n}=37)\end{array}$ & $\begin{array}{l}\text { Média } \\
(\mathrm{n}=16)\end{array}$ & $\begin{array}{l}\text { Grande } \\
(\mathrm{n}=8)\end{array}$ \\
\hline \multirow{9}{*}{ 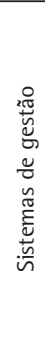 } & Aplicativos de administração e finanças & 99 & 93 & 100 & 100 & 100 \\
\hline & Aplicativos de vendas & 97 & 93 & 100 & 94 & 100 \\
\hline & Aplicativo para compras & 96 & 87 & 100 & 94 & 100 \\
\hline & Aplicativos para controle de estoques & 93 & 80 & 97 & 94 & 100 \\
\hline & Site na internet e comércio eletrônico & 82 & 100 & 70 & 94 & 75 \\
\hline & Gestão de relacionamento (CRM) & 34 & 27 & 30 & 44 & 50 \\
\hline & Gestão de cadeia de suprimentos (SCM) & 33 & 40 & 32 & 31 & 25 \\
\hline & Ligação com clientes e fornecedores (EDI) & 18 & 13 & 11 & 13 & 75 \\
\hline & Sistemas de informação gerencial (ESI e BI) & 37 & 20 & 30 & 56 & 63 \\
\hline \multirow{10}{*}{ 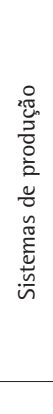 } & Planejamento dos recursos materiais (MRP) & 62 & 27 & 70 & 63 & 88 \\
\hline & Projeto auxiliado por computador (CAD) & 79 & 47 & 84 & 94 & 88 \\
\hline & Fabricação assistida por computador (CAM) & 36 & 7 & 41 & 38 & 63 \\
\hline & Sistemas para apoio ao controle de qualidade & 47 & 7 & 51 & 63 & 75 \\
\hline & $\begin{array}{c}\text { Sistemas avançados de planejamento e programação } \\
\text { de produção (APS) }\end{array}$ & 32 & 7 & 27 & 56 & 50 \\
\hline & Controle de fluxo de trabalho (Workflow) & 24 & 0 & 30 & 25 & 38 \\
\hline & Máquinas por controle numérico (CNC) & 53 & 0 & 70 & 56 & 63 \\
\hline & Controladores programáveis (PLC) & 33 & 0 & 35 & 44 & 63 \\
\hline & Robôs & 17 & 0 & 19 & 19 & 38 \\
\hline & Total & 54 & 36 & 55 & 60 & 70 \\
\hline
\end{tabular}

empresas que informaram utilizar cada um dos sistemas. A linha total indica um percentual médio do uso de sistemas, geral e por porte. Os resultados mostraram que as grandes empresas utilizam mais sistemas em comparação aos demais portes. A maior diferença de utilização deu-se entre pequenas e microempresas. Esses resultados estão de acordo com outros anteriormente obtidos (SOUZA et al., 2005) e indicam que o crescimento do grau de informatização de acordo com o porte também se verifica no setor estudado neste trabalho (bens de capital mecânicos).
Os resultados apontaram para uma predominância das grandes e médias empresas quanto à utilização dos sistemas de gestão e administração. No tocante aos sistemas de produção e projetos foi possível perceber que as porcentagens de empresas que os adotam são consideravelmente menor que as dos sistemas de gestão e administração. Os sistemas de produção e projetos são mais utilizados pelas empresas de maior porte. A utilização menor de softwares de produção (MRP, Controle de Qualidade, APS e Workflow) em relação aos sistemas de gestão e administração pode indicar 
maior interesse nas áreas administrativo-financeira, vendas e compras por parte das empresas brasileiras de bens de capital mecânicos.

Notou-se também que as empresas parecem dar mais atenção aos sistemas de projetos CAD que aos sistemas MRP, ou preferência por inovação em relação a otimizações de produção e de recursos materiais. lsso pode ser explicado pelo fato de boa parte das empresas fabricarem equipamentos sob encomenda, as quais têm menos necessidade de utilização de gerenciamentos ótimos de materiais e de sistemas modernos de produção como Just in Time. Da mesma forma, como os equipamentos são feitos sob solicitação, grande é a necessidade de utilização de sistemas CAD. Além disso, o CAD pode apresentar resultados mais imediatos e mais facilmente perceptíveis.

\subsubsection{Fatores críticos de sucesso (outputs da etapa intermediária)}

As questões ligadas ao estágio intermediário do modelo (item 3.1.2 e Quadro 4) apresentaram menor número de respondentes e mais respostas possivelmente inconsistentes que as demais partes do questionário. Isso já era esperado, uma vez que os dados solicitados nessa seção envolvem questões financeiras que as empresas normalmente hesitam em informar e muitas, principalmente as de menor porte, não conseguem responder adequadamente por falta de conhecimento ou mesmo por indisponibilidade da informação. Devido a isso, muitos indicadores de desempenho inicialmente previstos para compor o modelo precisaram ser descartados, e os fatores críticos de sucesso ficaram compostos da seguinte maneira conforme apresentado no Quadro 5.

\subsection{Aplicação da técnica DEA}

Para a análise dos dados com a técnica DEA foi utilizado o programa DEA-SAED V 1.0 (SURCO; WILHELM, 2004). Optou-se pela orientação a input, pois a eficiência dos resultados do uso da $\mathrm{Tl}$ de gestão e de produção é observada a partir dos inputs escolhidos, analisando-se como esses fatores auxiliam na transformação dos outputs. Foi também adotado o modelo BCC (ou VRS), que considera que um acréscimo no input poderá promover um acréscimo no output, não necessariamente proporcional. Belloni (2000) cita que o BCC avalia o conjunto de unidades dependendo da natureza dos rendimentos de escala que caracteriza o processo produtivo e permite comparar unidades de portes distintos. Conforme a amostra obtida, a diversidade de empresas é grande e há fortes indícios de não linearidade entre inputs e outputs. Como discutido no item 4.1.4, alguns indicadores para os fatores críticos de sucesso não puderam ser obtidos dos respondentes. Dessa forma, o sistema de análise de dois estágios originalmente mostrado na Figura 1 transformou-se no modelo mostrado na Figura 2.

Somente 28 empresas responderam a todos os dados necessários para execução do teste (8 microempresas, 15 pequenas, 3 médias e 2 grandes). Entretanto, isso não se tornou restritivo para a aplicação da técnica, pois se sugere que o número de DMUs deve ser no mínimo igual ao dobro do número total de variáveis utilizadas, inputs mais outputs, no modelo adotado (LINS; MEZA, 2000), embora, claro, isso traga problemas para a generalização dos resultados. A partir dos dados das 28 empresas foram realizadas três análises com resultados apresentados na Tabela 4: a primeira considerando as entradas do primeiro estágio e as saídas do segundo estágio ("global”); a segunda considerando as entradas e saídas do primeiro estágio; e a terceira considerando as entradas e saídas do segundo estágio. 0 modelo global analisa a capacidade das empresas converterem investimentos e despesas em $\mathrm{Tl}$ diretamente em lucratividade, enquanto os dois modelos testam a conversão em cada um dos estágios.

Em cada grupo de colunas da Tabela 4, a primeira (Clas.) representa a classificação de cada empresa, conforme o índice de produtividade (Ind. DEA), lembrando que o índice representa a produtividade da empresa em relação a empresas situadas na fronteira de eficiência, com valores

Quadro 5. Fatores críticos de sucesso obtidos e seus indicadores.

\begin{tabular}{|c|c|c|c|c|c|c|}
\hline $\begin{array}{c}\text { Fatores críticos } \\
\text { de sucesso }\end{array}$ & Indicadores de desempenho & Micro & Pequenas & Médias & Grandes & Todas \\
\hline Qualidade & Qualidade percebida & 4.18 & 4.30 & 4.15 & 4.71 & 4.28 \\
\hline Confiabilidade & Taxa de atrasos $(\%)$ & 12.78 & 22.53 & 21.81 & 9.13 & 18.9 \\
\hline Produtividade & Faturamento no ano de 2007/número de funcionários & 274.774 & 165.893 & 370.801 & 342.501 & 233.056 \\
\hline Flexibilidade & Flexibilidade da mão de obra $(\%)$ & 40.12 & 28.14 & 17.64 & 47.5 & 30.89 \\
\hline lnovação & Número de novos produtos & 7.14 & 2.94 & 3.60 & 18.00 & 5.21 \\
\hline Eficácia & Número de horas extras trabalhadas & $1.183,75$ & $3.282,68$ & $7.453,62$ & $22.412,67$ & $4.465,00$ \\
\hline
\end{tabular}




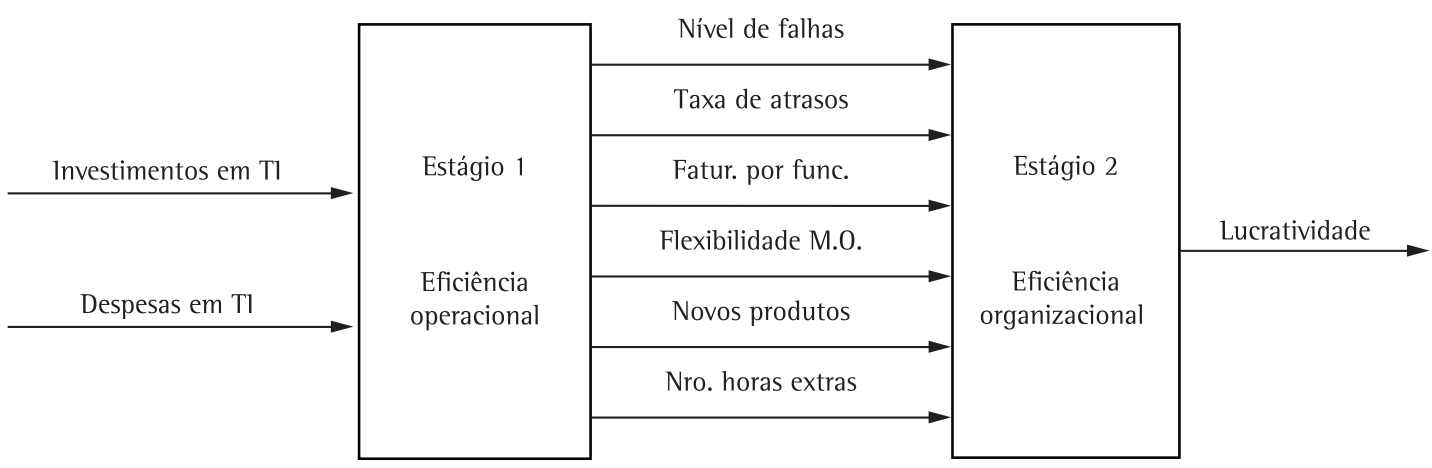

Figura 2. Modelo de eficiência executado a partir dos dados obtidos na amostra.

Tabela 4. Resultado da aplicação da técnica DEA.

\begin{tabular}{|c|c|c|c|c|c|c|c|c|c|c|c|}
\hline \multicolumn{4}{|c|}{ Global } & \multicolumn{4}{|c|}{ Primeiro estágio } & \multicolumn{4}{|c|}{ Segundo estágio } \\
\hline Clas. & Empr. & Porte & Índice DEA & Clas. & Empr. & Porte & Índice DEA & Clas. & Empr. & Porte & Índice DEA \\
\hline 1 & EMP2 & 1 & 1,000 & 1 & EMP1 & 1 & 1,000 & 1 & EMP3 & 1 & 1,000 \\
\hline 1 & EMP6 & 1 & 1,000 & 1 & EMP2 & 1 & 1,000 & 1 & EMP4 & 1 & 1,000 \\
\hline 1 & EMP9 & 2 & 1,000 & 1 & EMP3 & 1 & 1,000 & 1 & EMP6 & 1 & 1,000 \\
\hline 1 & EMP10 & 2 & 1,000 & 1 & EMP4 & 1 & 1,000 & 1 & EMP8 & 1 & 1,000 \\
\hline 1 & EMP16 & 2 & 1,000 & 1 & EMP5 & 1 & 1,000 & 1 & EMP9 & 2 & 1,000 \\
\hline 1 & EMP20 & 2 & 1,000 & 1 & EMP6 & 1 & 1,000 & 1 & EMP12 & 2 & 1,000 \\
\hline 1 & EMP21 & 2 & 1,000 & 1 & EMP7 & 1 & 1,000 & 1 & EMP14 & 2 & 1,000 \\
\hline 1 & EMP23 & 2 & 1,000 & 1 & EMP11 & 2 & 1,000 & 1 & EMP15 & 2 & 1,000 \\
\hline 1 & EMP24 & 3 & 1,000 & 1 & EMP16 & 2 & 1,000 & 1 & EMP16 & 2 & 1,000 \\
\hline 1 & EMP27 & 4 & 1,000 & 1 & EMP17 & 2 & 1,000 & 1 & EMP17 & 2 & 1,000 \\
\hline 1 & EMP28 & 4 & 1,000 & 1 & EMP21 & 2 & 1,000 & 1 & EMP18 & 2 & 1,000 \\
\hline 12 & EMP8 & 1 & 0,938 & 1 & EMP27 & 4 & 1,000 & 1 & EMP19 & 2 & 1,000 \\
\hline 13 & EMP7 & 1 & 0,756 & 1 & EMP28 & 4 & 1,000 & 1 & EMP20 & 2 & 1,000 \\
\hline 14 & EMP3 & 1 & 0,702 & 14 & EMP8 & 1 & 0,907 & 1 & EMP21 & 2 & 1,000 \\
\hline 15 & EMP5 & 1 & 0,626 & 15 & EMP23 & 2 & 0,353 & 1 & EMP22 & 2 & 1,000 \\
\hline 16 & EMP26 & 3 & 0,484 & 16 & EMP12 & 2 & 0,148 & 1 & EMP23 & 2 & 1,000 \\
\hline 17 & EMP22 & 2 & 0,336 & 17 & EMP10 & 2 & 0,137 & 1 & EMP24 & 3 & 1,000 \\
\hline 18 & EMP25 & 3 & 0,294 & 18 & EMP19 & 2 & 0,125 & 1 & EMP25 & 3 & 1,000 \\
\hline 19 & EMP19 & 2 & 0,257 & 19 & EMP22 & 2 & 0,123 & 1 & EMP26 & 3 & 1,000 \\
\hline 20 & EMP1 & 1 & 0,250 & 19 & EMP26 & 3 & 0,123 & 1 & EMP27 & 4 & 1,000 \\
\hline 21 & EMP4 & 1 & 0,243 & 21 & EMP18 & 2 & 0,077 & 1 & EMP28 & 4 & 1,000 \\
\hline 22 & EMP12 & 2 & 0,196 & 22 & EMP13 & 2 & 0,067 & 22 & EMP10 & 2 & 0,966 \\
\hline 23 & EMP11 & 2 & 0,184 & 23 & EMP9 & 2 & 0,063 & 23 & EMP7 & 1 & 0,919 \\
\hline 24 & EMP15 & 2 & 0,162 & 23 & EMP14 & 2 & 0,063 & 24 & EMP5 & 1 & 0,812 \\
\hline 25 & EMP17 & 2 & 0,156 & 25 & EMP15 & 2 & 0,056 & 25 & EMP1 & 1 & 0,800 \\
\hline 26 & EMP18 & 2 & 0,118 & 26 & EMP20 & 2 & 0,016 & 25 & EMP2 & 1 & 0,800 \\
\hline 27 & EMP13 & 2 & 0,104 & 27 & EMP24 & 3 & 0,015 & 25 & EMP11 & 2 & 0,800 \\
\hline 28 & EMP14 & 2 & 0,069 & 28 & EMP25 & 3 & 0,005 & 25 & EMP13 & 2 & 0,800 \\
\hline
\end{tabular}

que vão de zero (totalmente ineficientes) a um (eficientes). Empresas com mesmo valor de índice obtiveram a mesma classificação. Para não citar o nome da empresa designou-se um número para cada uma delas, precedido pelas letras EMP conforme a segunda coluna (Empr.). A terceira coluna mostra o porte da empresa, sendo 1 o número utilizado para microempresas, 2 para pequenas, 3 para médias e 4 para grandes.
Nota-se uma grande variação nos resultados das diversas empresas no sistema global e no primeiro estágio, enquanto o índice de eficiência varia pouco no segundo. De maneira geral, as empresas mostraram-se mais eficientes no segundo estágio do que no primeiro. No segundo estágio as empresas encontram-se bastante concentradas nos índices superiores, principalmente na fronteira de eficiência (21 empresas) daquele estágio. Isso poderia indicar 
que, de fato, os indicadores selecionados para avaliação de fatores críticos de sucesso (obtidos de acordo com a literatura) estão relacionados à lucratividade das empresas neste determinado setor. Entretanto, devido ao tamanho da amostra obtida, essa é uma afirmação que exige cuidado e novo trabalho de campo. Uma menor quantidade de empresas mostrou-se eficiente em converter os investimentos e despesas em $\mathrm{Tl}$ em fatores críticos de sucesso, no primeiro estágio, em que há 13 empresas na fronteira de eficiência, sendo que mais 13 estão abaixo do índice 0,148 . Isso pode ser mais uma indicação de que a $\mathrm{Tl}$, nessas empresas, tem sido pouco aplicada visando melhorias diretas nos fatores críticos de sucesso dessas empresas, mais voltadas a sistemas administrativos e apoios (como evidenciado no item 4.1.3). Isso traz reflexo direto para o modelo testado.

Quanto ao porte, 25\% das microempresas classificaram-se na fronteira eficiente global, 87\% na fronteira eficiente do primeiro estágio e 50\% na do segundo estágio. Das empresas pequenas, $40 \%$ na fronteira eficiente global, $27 \%$ na do primeiro estágio e $80 \%$ na do segundo. Entre as empresa médias, 33\% na global, 0\% na do primeiro estágio e 100\% no segundo estágio. As empresas grandes foram as que melhor desempenharam, com 100\% delas nas três fronteiras eficientes. Há de se considerar que tivemos apenas três empresas médias e duas grandes na amostra de 28 empresas analisadas pela DEA. Considerando-se apenas o subsetor de máquinas-ferramenta, apenas 14 empresas da amostra puderam ser utilizadas, dessa maneira optou-se por não incluir os resultados da análise do subsetor neste trabalho.

\subsection{Correlações entre o índice de eficiência e os fatores de informatização}

Com os índices de eficiência obtidos no item 4.2 analisaram-se suas correlações com os fatores de informatização (grau de informatização - Gl, integração tecnológica e organizacional - IT0 e condições de operação - CO) e com o porte da empresa (número de 1 a 4) utilizando-se o software SPSS v.15. Para os fatores de informatização foi utilizada a correlação de Pearson, adequada para variáveis métricas, e, para o porte da empresa, a correlação de Spearman, adequada para variáveis ordinais. Os resultados encontrados são mostrados na Tabela 5. Por conta do tamanho da amostra, considerou-se o nível de 5\% e 10\% para o teste da significância das correlações.

Como se pode observar na Tabela 5, no modelo global não foram encontradas correlações positivas
Tabela 5. Correlações significativas entre índice de eficiência e fatores de informatização.

\begin{tabular}{cccccc}
\hline $\begin{array}{c}\text { Indice } \\
\text { DEA }\end{array}$ & $(\mathrm{n}=28)$ & Gl & ITO & CO & Porte \\
\hline \multirow{2}{*}{ Global } & coef. correlação & $-0,018$ & $-0,146$ & $-0,104$ & 0,076 \\
& sig. (bicaudal) & 0,926 & 0,459 & 0,598 & 0,697 \\
\cline { 2 - 6 } Primeiro & coef. correlação & 0,345 & 0,465 & 0,318 & $-0,455$ \\
estágio & sig. (bicaudal) & 0,073 & 0,013 & 0,099 & 0,014 \\
\cline { 2 - 6 } Segundo & coef. correlação & 0,036 & 0,046 & $-0,279$ & 0,395 \\
estágio & sig. (bicaudal) & 0,854 & 0,816 & 0,150 & 0,037 \\
\hline
\end{tabular}

ou negativas dentro de um nível de significância de $10 \%$ entre o índice DEA global e os diversos fatores de informatização. Isso poderia indicar que os aspectos ligados à gestão e à adequada utilização da Tl não estão relacionados a uma melhor obtenção de resultados por meio de sua aplicação, o que seria uma contradição com boa parte da literatura. Entretanto, essa ausência de correlação pode ser analisada observando-se os resultados a partir dos dois estágios, separadamente.

Em relação ao primeiro estágio, as correlações com os três fatores de informatização mostraram-se significativas a um nível de $10 \%$ (Gl e CO) e ao nível de 5\% (IT0 e porte). Isso indica que, como esperado, as empresas com maior combinação de grau de informatização, integração organizacional e condições de operação tendem a ser mais eficientes dentro do processo de conversão estabelecido para o primeiro estágio, favorecendo, portanto, a melhora dos FCS qualidade, flexibilidade, inovação e eficácia. Ou seja, o planejamento e a gestão de $\mathrm{Tl}$ contribuem para sua correta aplicação nas empresas. 0 fator mais fortemente correlacionado foi o de integração organizacional, justamente aquele que implica no alinhamento da organização à integração proporcionada pelo uso de sistemas.

Entretanto, no segundo estágio, os fatores de informatização não estão correlacionados ao índice de eficiência. lsso pode indicar que a transformação dos FCS obtidos em lucratividade depende de fatores além do uso correto da Tl, o que faz bastante sentido. Por exemplo, aspectos como estratégia empresarial, concorrência, correto posicionamento, entre outros, trariam mais impacto a essa conversão do que a $\mathrm{Tl}$ (que, sem dúvida, precisa estar corretamente instalada e ser adequadamente utilizada para possibilitar que os FCS estejam disponíveis para poderem ser transformados em resultados - é esse o papel da $\mathrm{Tl}$ ).

Note que a correlação entre o índice de eficiência do primeiro estágio e o porte é negativa, enquanto que no segundo estágio a correlação com o porte mostrou-se significativa, também ao 
nível de 5\%. Isso pode significar que as empresas menores podem ter mais eficiência em melhorar os $\mathrm{FCS}$, otimizando as despesas em $\mathrm{Tl}$, investimento em $\mathrm{Tl}$ e melhorando a confiabilidade. lsso pode ser explicado porque as empresas menores têm mais agilidade para atender à qualidade, flexibilidade, inovação e eficácia. Por outro lado, pode significar que as empresas maiores são mais efetivas em transformar os FCS em lucratividade e faturamento. Isso faz sentido porque as empresas menores, conforme La Rovere (2001), possuem de forma geral baixa capacidade gerencial, apesar de serem mais ágeis. Além disso, muitas vezes o porte e a escala permitem às grandes empresas obter vantagens de negociação, independentemente de outros fatores críticos de sucesso.

\section{Conclusões}

Este estudo analisou a relação entre o uso da $\mathrm{Tl}$ e a eficiência das empresas do setor brasileiro de bens de capital mecânicos, e o papel dos fatores de informatização nessa relação. Para verificar a eficiência das empresas foi utilizada a técnica DEA, que estabelece um grau crescente de zero a um indicando a eficiência das empresas pesquisadas, conforme sua capacidade de transformar inputs em outputs. Os inputs, que incluem despesas em $\mathrm{Tl}$ e investimentos em $\mathrm{Tl}$, e outputs, encontrados através de pesquisa bibliográfica sobre os fatores críticos de sucesso das empresas de bens de capital, seguindo metodologia sugerida por Francischini (1998). Para poder observar os processos intermediários aplicou-se o método DEA para dois estágios, e para atender a uma amostra heterogênea adotou-se a técnica DEA modelo BCC. Em seguida foram comparados os índices de eficiência com os fatores de informatização.Verificou-se uma hegemonia das maiores empresas no tocante ao uso da Tl e seu gerenciamento. Apesar de elas gastarem e investirem mais em $\mathrm{Tl}$, possuem menores relações despesas em $\mathrm{Tl} /$ faturamento e investimento $\mathrm{em} \mathrm{Tl} /$ faturamento, o que lhes permite obter vantagem financeira relativa para melhorar os seus sistemas de informação. 0 porte da empresa, portanto, pode ser colocado como o principal fator de diferenciação encontrado neste trabalho.

As empresas grandes mostraram-se eficientes nas três etapas: na etapa geral de conversão de variáveis de $\mathrm{Tl}$ em rentabilidade, no primeiro estágio que converte variáveis de $\mathrm{Tl}$ em fatores críticos de sucesso e no segundo estágio que converte os FCS em rentabilidade. Apesar de não se ter concluído sobre a relação entre a utilização da $\mathrm{Tl}$ e a rentabilidade das empresas, observou-se haver correlação entre os índices de eficiência do primeiro estágio com os principais fatores de informatização, permitindo conjecturar-se sobre a influência do grau de informatização, condições de operação de Tl e integração organizacional nos fatores críticos de sucesso adotados. Verificou-se que as empresas menores podem ser mais eficientes em melhorar os FCS através do uso da Tl, porém as maiores parecem ser mais eficientes na conversão dos FCS em rentabilidade, possivelmente por seu melhor gerenciamento e por questões de escala.

A grande limitação encontrada para a realização desta pesquisa foi dificuldade de obtenção das informações das empresas envolvidas, em sua maioria micro e pequenas, o que resultou em um número reduzido de questionários respondidos total ou parcialmente, e uma consequente amostra reduzida para a realização da DEA. Embora a técnica permita esse tamanho de amostra, há menor possibilidade de generalização de resultados. Outro aspecto foi a impossibilidade de manter os custos da empresa como entrada para o modelo, o que eliminou a possibilidade de verificar se empresas em que os fatores de informatização estão presentes são também capazes de transformar investimentos e despesas em $\mathrm{Tl}$ em redução de custos.

\section{Referências}

AHUMADA-LOBO, 1. M. Three essays on labor economics: the equilibrium job search approach. Tese (Doutorado)Carnegie Mellon University, Pittsburgh, 2002.

AVELLAR, A. P. Relatório setorial preliminar. Brasília: FINEP 2007. Disponível em: <www.finep.gov.br>. Acesso em: 20 ago. 2007.

BECCHETTI, L. ICT investment, productivity, and efficiency: evidence at firm level using a stochastic frontier approach. Journal of Productivity Analysis, n. 20, p. 143-167, 2003. http://dx.doi.org/10.1023/A:1025128121853

BELLONI, J. A. Uma metodologia de avaliação da eficiência produtiva de universidades federais brasileiras. Tese (Doutorado)-Universidade Federal de Santa Catarina, Florianópolis, 2000.

BUONANNO, G.; GRAMIGNOLI, S; RAVARINI, A. ICT diffusion and strategic role within ltalian SMEs. In: TAN, F (Org.). Global perspective of information technology management. Hershey: ldea Group, 2002.

CORRÊA, H. L.; CORRÊA, C. A. Administração de produção e operações. São Paulo: Atlas, 2005.

DASGUPTA,S.;SARKIS, J.;TALLURI, S. Influence of information technology on firm productivity: a cross-sectional study. Logistics Information Management, v. 12, p. 120-129, 1999. http://dx.doi.org/10.1108/09576059910256493

FRANCISCHINI, P. G. Aplicação do modelo de FCS para a obtenção de indicadores de produtividade. In: ENCONTRO NACIONAL DE ENGENHARIA DE PRODUÇÃO - ENEGEP, 1998, 1998, Niterói, Rio de Janeiro. Anais... Rio de Janeiro: ABEPRO, 1998. 
HITT, L.; BRYNJOLFSSON, E. The three faces of IT value: theory and evidence. In: INTERNATIONAL CONFERENCE ON INFORMATION SYSTEMS, 15., 1994, Vancouver. Proceedings... p. 263-377.

KUDYBA, S.; DIWAN, R. Information technology, corporate productivity and the new economy. Londres: Quorum Books, 2002

LA ROVERE, R. L. Perspectivas das micro, pequenas e médias empresas no Brasil. Revista de Economia Contemporânea, Rio de Janeiro, 2001. Edição especial.

LAGACÉ, D. Succès d'implantation des technologies manufacturières avancées. Tese (Doutorado)-École Polytechnique de Montréal, Montreal, 2000.

LEE, B.; BARUA, A. An integrated assessment of productivity and efficiency impacts of information technology investments: old data, new analysis and evidence. Journal of Productivity Analysis, v. 12, n. 1, p. 21-43, 1999. http://dx.doi.org/10.1023/A:1007898906629

LINS, M. P.; MEZA, L. A. Análise envoltória de dados e perspectivas de integração no ambiente de apoio à decisão. Rio de Janeiro: COPPE; UFRJ, 2000.

MAÇADA, A. C.; BECKER, J.; LUNARDI, G. L. Efetividade de conversão dos investimentos em $\mathrm{Tl}$ na eficiência dos bancos brasileiros. Revista de Administração Contemporânea, v. 9, n. 1, p. 9-33, 2005.

MELLO, A. S. A política industrial para o setor de bens de capital. 2004. Disponível em: <www.redmercosur.org>. Acesso em: 30 ago. 2007.

MENDES, J. V.; ESCRIVÃO FILHO, E. Sistemas integrados de gestão em pequenas e médias empresas: um confronto entre o referencial teórico e a prática empresarial. Gestão e Produção, v. 9, n. 3, p. 277-296, 2002.

MUSCAT, A.; FLEURY, A. Indicadores de qualidade e produtividade na indústria brasileira. Revista Indicadores de Qualidade e Produtividade, v. 1, n. 2, p. 82-87, 1993.

PETRONI, A.; BEVILACQUA, M. Identifying manufacturing flexibility best practices in small and medium enterprises. International Journal of Operations \& Production
Management, Bradford, v. 22, n. 7-8, p. 929-948, 2002. http://dx.doi.org/10.1108/01443570210436217

RAI, A.; PATNAYAKUNI, R.; PATNAYAKUNI, N. Technology investment and business performance. Communication of the ACM, v. 40, n. 7, p. 89-97, 1997. http://dx.doi. org/10.1145/256175.256191

SERSON, S. M. Fábrica veloz: um modelo para competir com base no tempo. Dissertação (Mestrado)-Universidade de São Paulo, São Paulo, 1996.

SINK, D. S.; TUTTLE, T. C. Planejamento e medição para a performance. Rio de Janeiro: Qualitymark, 1993.

SOUZA, C. A. et al. Avaliação do grau de informatização de empresas: um estudo em indústrias brasileiras. In: ENCONTRO DA ASSOCIAC̄̃̃O NACIONAL DOS PROGRAMAS DE PÓS-GRADUAÇÃO EM ADMINISTRAÇÃO - EnANPAD, 29., 2005, Brasília. Anais... Brasília: ANPAD, 2005. CD ROM.

SURCO, D. F.; WILHELM, V. E. DEA-SAED: software de análises envoltória de dados. Curitiba: Universidade Federal do Paraná, 2004.

TIRONI, L. F. Indicadores da qualidade e produtividade: conceitos e usos. Revista Indicadores da Qualidade e Produtividade do Instituto de Pesquisa Econômica Aplicada, n. 1, 1993. Artigo 1.

VALENTE, N. T. Z.; RICCIO, E. L. Implementação de ERPs em pequenas e médias empresas: aspectos relevantes apontados pela literatura. In: CONGRESSO NACIONAL DE GESTÃO DA TECNOLOGIA E SISTEMAS DE INFORMAÇÃO, 1., 2004, São Paulo. Anais... São Paulo: TECSI, 2004.

WANG, C. H.; GOPAL, R. D.; ZIONTS, S. Use of data envelopment analysis in assessing information technology impact on firm performance. Annals of Operations Research, v. 73, p. 191-213, 1997. http:// dx.doi.org/10.1023/A:1018977111455

WEILL, $P$. The relationship between investment in information technology and firm performance: a study of the valve manufacturing sector. Information Systems Research, v. 3, n. 4, p. 307-333, 1992. http://dx.doi.org/10.1287/ isre.3.4.307

\title{
Information Technology and organizational efficiency: a study in the brazilian capital goods sector focusing on micro, small and medium enterprises
}

\begin{abstract}
The impact on companies' performance is among the possible measures for Information Technology (IT) success. Several researches showing the influence of IT on firms' results have been conducted, but mainly through studies carried out in large enterprises. The objective of this work was to analyze the relationship between IT investments and organizational efficiency - focusing on micro, small and medium sized enterprises. To this end, critical success factors for industrial companies' performance were identified, and a two-stage data envelopment analysis (DEA) model was developed and tested in a sample of firms in the capital goods sector. DEA is especially interesting because it allows comparing and differentiating the firms in the sample which are more efficient in deriving results from IT. Among the results observed, there were the higher capacity of small firms to transform IT investments into operational efficiency and the higher capacity of larger companies to convert critical success factors into profitability.
\end{abstract}

Keywords

Information technology. Computerization. Manufacturing industries. Micro, small and medium enterprises management. Data Envelopment Analysis - DEA. 